\title{
Atom-Probe Tomography: Detection Efficiency and Resolution of Nanometer-Scale Precipitates in a Ti-5553 Alloy
}

D. Isheim ${ }^{1,2}$, J. Coakley $^{1,3}$, A. Radecka ${ }^{4,5}$, D. Dye ${ }^{4}$, T.J. Prosa ${ }^{6}$, Y. Chen ${ }^{6}$, P.A.J. Bagot ${ }^{7}$ and D.N. Seidman ${ }^{1,2}$

${ }^{1}$ Department of Materials Science and Engineering, Northwestern University, Evanston, IL, USA

${ }^{2}$ Center for Atom-Probe Tomography (NUCAPT), Northwestern University, Evanston, IL, USA

${ }^{3}$ Department of Materials Science and Metallurgy, University of Cambridge, Cambridge, England

${ }^{4}$ Department of Materials, Imperial College, South Kensington, London, England

${ }^{5}$ Rolls Royce Plc, Elton Road, Derby, England

${ }^{6}$ CAMECA Instruments, Inc., Madison, WI, USA

${ }^{7}$ Department of Materials, University of Oxford, Oxford, England

Atom-probe tomography (APT) is a characterization technique for materials with nano-scale compositional variations or precipitates with subnanometer spatial resolution in $3 \mathrm{D}$ and analytical resolution of typically $\sim 10$ at.ppm [1-3]. Applications include the early stages of phase transformations, precipitation in metals and alloys as well as the study of nano-scale features in biominerals, semiconductor devices, and geological samples. Most APTs in use today employ a microchannel plate (MCP) as the primary detector with single-atom sensitivity. The detection efficiency of an MCP is, however, limited by the open-area ratio of approximately 55\% [1]. The open-area ratio affects the detection of all elements in the same way and therefore quantitative compositions can be determined by simply counting atoms. Including additional losses in signal processing and limitations with multi-hit deconvolution, the total system detection efficiency of an APT with a straight flight path typically is approximately $50 \%$. Limitations arising from the limited detection efficiency for the statistically significant resolution of nano-scale features with small compositional variations, sometimes termed "clusters," have long been recognized and modeling predicts that detection of nano-scale features can be significantly improved with increased detection efficiencies $[4,5,6]$. Recently, first results obtained with a prototype APT with an estimated $\sim 80 \%$ system detection efficiency have been published [6]. A recent quantitative determination of the total system detection efficiency of that APT system resulted in 77 $\pm 2 \%$ [7].

To test the impact of an increased detection efficiency on resolving nano-scale features, we used the test case of small ( $\sim 1-2 \mathrm{~nm}$ radius) Ti-enriched precipitates in a titanium alloy, Ti-5Al-5Mo-5V-3Cr- $0.3 \mathrm{Fe}$ wt.\% (Ti-9Al-3Mo-5V-3Cr-0.3Fe at.\%) [8], known commercially as Ti-5553, which is utilized in the Boeing 787 airframes as large section forgings [9]. Ti-rich $\omega$-precipitates have been detected in this alloy after a $1 \mathrm{~h}$ aging treatment at $300^{\circ} \mathrm{C}$ by small-angle neutron scattering (SANS) and transmission electron microscopy (TEM) [9] but the precise morphology, dispersion, and specifically the composition of these precipitates remains unknown. We employed a CAMECA LEAP 4000X Si with 50\% detection efficiency, and a CAMECA LEAP 5000 XS with 80\% nominal efficiency to study these precipitates. APT tips were prepared using a standard lift-out, mounting, and tip-sharpening method [10] utilizing an FEI Helios NanoLab 600 DualBeam $^{\mathrm{TM}}$ focused ion-beam (FIB) microscope equipped with an Omniprobe $^{\mathrm{TM}}$ micromanipulator. APT analyses were conducted on both instruments with identical run conditions, $15 \mathrm{pJ}$ pulse energy with ultraviolet (355 nm wavelength) laser pulses, $250 \mathrm{kHz}$ pulse repetition rate and at a specimen stage temperature of $30 \mathrm{~K}$. The target detection rate was set at $2 \%$ for the LEAP 4000X Si and 3.2\% for the LEAP $5000 \mathrm{XS}$, taking into account the different detection efficiencies. These conditions resulted in charge-state peak intensity ratios $(\mathrm{CSRs})$ of $\mathrm{Al}^{++} / \mathrm{Al}^{+}=9.1$ and 
1.2, and 556 and 1650 for $\mathrm{Ti}^{++} / \mathrm{Ti}^{3+}$ for the $4000 \mathrm{X} \mathrm{Si}$ and the $5000 \mathrm{XS}$ datasets, respectively, indicating a slightly higher effective temperature for laser-assisted field-evaporation for the 4000X Si instrument. Data reconstruction and evaluation were performed utilizing CAMECA's IVAS 3.6.12 software.

The LEAP 5000 XS resolves a dense dispersion of small Ti-rich precipitates, Fig. 1(a), delineated by an 86 at.\% Ti isoconcentration surface. In contrast, precipitates cannot be distinguished from random concentration fluctuations in the data acquired with the $4000 \mathrm{X} \mathrm{Si}$, Fig. 1(b). To ensure the statistical significance of the precipitates detected in the $5000 \mathrm{XS}$ dataset, concentration frequency distributions were calculated with a block size of $\mathrm{N}=160$ atoms for the 5000 XS data and $\mathrm{N}=100$ atoms for the 4000X Si data, representing sampling volumes of the same size, taking into account the different detection efficiencies of both instruments, and compared with binomial distributions representing a homogeneous alloy with the same mean composition. The concentration frequency distribution for the 5000 XS data, Fig. 1(c), has a significantly enhanced tail at higher Ti concentrations (arrow), and concomitantly a smaller maximum value, indicating the presence of precipitates, whereas the 4000X Si distribution, Fig. 1(d) is indistinguishable from the random case. The higher detection efficiency of the $5000 \mathrm{XS}$ is critical for resolving nanometer-scale features that are either small in size and/or have a small compositional amplitude.

\section{References:}

[1] B Gault et al, “Atom Probe Microscopy”, Springer, NewYork, 2012.

[2] DJ Larson et al, "Local Electrode Atom Probe Tomography: A User's Guide", Springer, 2014.

[3] DN Seidman and K Stiller, MRS Bulletin 34 (2009), p. 717.

[4] AV Ceguerra et al, Acta Crystallographica A68 (2012) p. 547.

[5] LT Stephenson et al, Philosophical Magazine 93(8) (2013) p. 975.

[6] MK Miller et al, Journal of Nuclear Materials 462 (2015) p. 428.

[7] TJ Prosa et al, Proc. of SPIE 9173 (2014) p. 917307-1.

[8] J Coakley et al, Journal of Alloys and Compounds 646 (2015) p. 946.

[9] RR Boyer and RD Briggs, Journal of Materials Engineering and Performance 14 (2005) p. 681.

[10] K Thompson et al, Ultramicroscopy 107 (2007) p.131.

[11] The LEAP 4000X Si atom-probe tomograph at the NUCAPT facility of Northwestern University was acquired and upgraded with equipment grants from the MRI program (NSF DMR-0420532) and the DURIP program of the Office of Naval Research (N00014-0400798, N00014-0610539, N000140910781). NUCAPT received support from the MRSEC program (NSF DMR-1121262) at the Materials Research Center, SHyNE Resource (NSF NNCI-1542205), and the Initiative for Sustainability and Energy (ISEN) at Northwestern University.
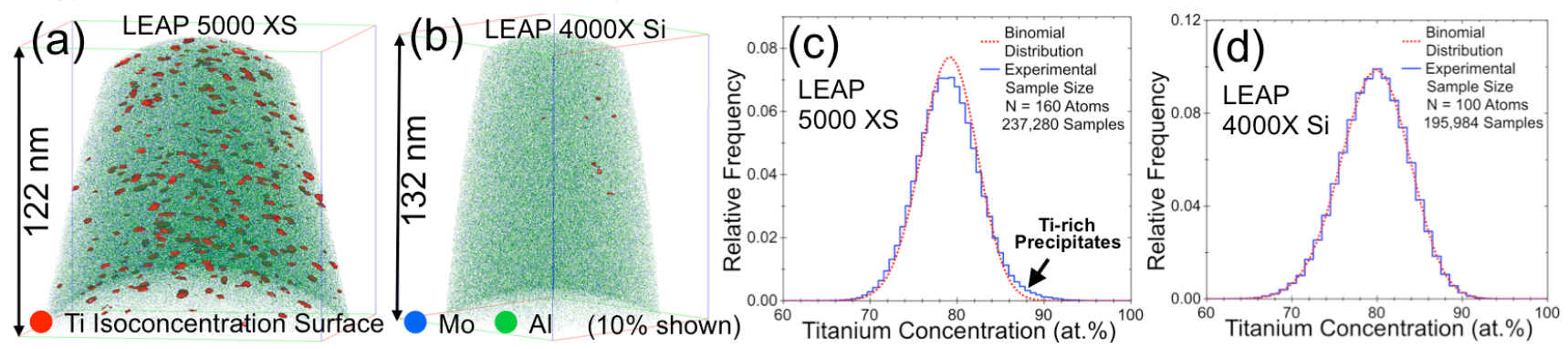

Figure 1: Impact of increased $80 \%$ reconstruction efficiency with the LEAP 5000 XS: The 86 at.\% Ti isoconcentration surface (a) and concentration frequency distribution (c) clearly show the presence of small Ti-rich precipitates with $\sim 1-2 \mathrm{~nm}$ radius in a Ti-5553 alloy aged at $300^{\circ} \mathrm{C}$ for $1 \mathrm{~h}$, whereas the LEAP 4000X Si data does not resolve the precipitates, (b), (d). 